\title{
SENSORIAMENTO REMOTO EM ECOLOGIA DA PAISAGEM: ESTADO DA ARTE
}

\author{
REMOTE SENSING IN THE LANDSCAPE ECOLOGY: STATE OF THE ART \\ Hugo José RIBEIRO, Nilson Clementino FERREIRA, Kátia Alcione KOPP, Tatiane Souza \\ Rodrigues PEREIRA, Wellington Nunes de OLIVEIRA \\ Universidade Federal de Goiás. Setor Leste Universitário, CEP: 74605-220 - Goiânia - GO. \\ Emails: hgribeirogeo@gmail.com; nclferreira@gmail.com; kakopp@gmail.com; tatiane.srp@hotmail.com; \\ wellington.wno@gmail.com

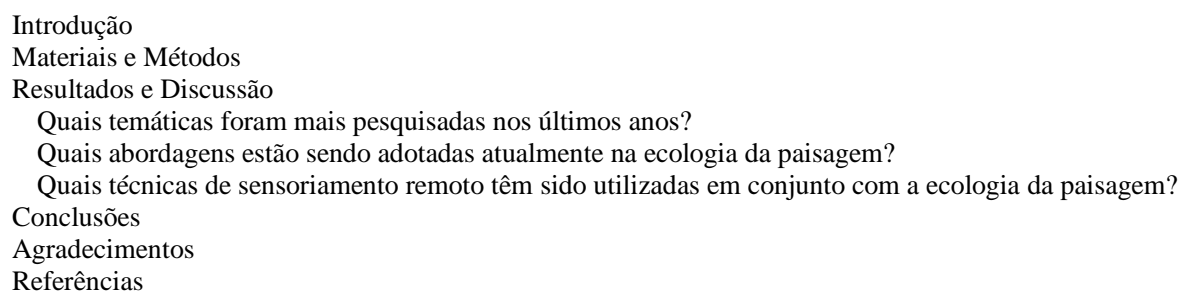

RESUMO - Os estudos de ecologia da paisagem, assim como os padrões espaciais, influenciam os processos ecológicos e, a utilização desta informação com a cobertura terrestre por detecção remota é indispensável para a realização de análises espaciais. Este artigo de revisão discute como o Sensoriamento Remoto está sendo utilizado em Ecologia da Paisagem nos últimos sete anos. Para isso, foi feita uma revisão sistemática da literatura com o apoio do programa State of the Art - START projetado especificamente para este propósito, considerando artigos de periódicos indexados com fator de impacto maior que dois. A pesquisa foi realizada a partir de questões, como: quais tópicos são mais pesquisados nos últimos anos? Que tipo de abordagem está sendo aplicada? E quais técnicas de sensoriamento remoto (SR) estão sendo usadas na pesquisa de ecologia da paisagem? Essas perguntas foram respondidas pela vasta literatura científica disponível. A pesquisa mostrou que os tópicos mais pesquisados foram o padrão e dinâmica espacial, ambos com cerca de $24 \%$ dos artigos analisados. A abordagem metodológica mais utilizada pelos pesquisadores com $61 \%$ do total e o pré-processamento foi a técnica mais amplamente aplicada, com $81 \%$ do total.

Palavras-chave: sensoriamento remoto, processamento de imagens, ecologia da paisagem, START, classificação.

\begin{abstract}
The studies landscape ecology how spatial patterns influence ecological processes and to use this information and land cover from remote sensing are indispensable for carrying the out analyzes. This review article discusses how Remote Sensing was used in Landscape Ecology in the last five years. For this was made a systematic review of the literature with supporting the program State of the Art - START designed specifically for this purpose, considering journal articles indexed with an impact factor greater than 2. The survey was conducted from questions such as what topics more are being researched in recent years? What kind of approach is being applied? And what remote sensing techniques (SR) are being used in landscape ecology research? These questions were answered by the vast scientific literature available. Research has shown that the most searched topics were spatial pattern and dynamics, both with about $24 \%$ of the full analyzed articles. The methodological approach was the most used by researchers with $61 \%$ of the total and the preprocessing was the most widely applied technique with $81 \%$ of the total.
\end{abstract}

Keywords: remote sensing, image processing, landscape ecology, START, classification.

\section{INTRODUÇÃO}

A ecologia da paisagem é uma ciência relativamente nova introduzida pelo biogeógrafo alemão Carl Troll em 1940. Com enfoque geográfico e influências da escola europeia, esse autor determinou o conceito básico de paisagem: para Troll (1971) "a noção básica de paisagem é a espacialidade, a heterogeneidade do espaço onde o homem habita". O foco de estudo desta escola de pensamento é o planejamento da ocupação territorial com base nas relações antrópicas nas unidades de paisagem em expressivas amplitudes geográficas (Metzger, 2001).

Na década de 1980 os pesquisadores norte- americanos adotaram uma abordagem de priorização das espécies para a ecologia da paisagem. Neste contexto o foco era direcionado às espécies da fauna e da flora, onde o mosaico de ecossistemas representa um conjunto de habitats que oferece condições mais favoráveis ou não para diferentes comunidades pesquisadas. Desse modo, a paisagem passou a ser priorizada pelas espécies existentes nos vários habitats, e a escala geográfica de trabalho nesta abordagem não necessariamente precisava ser ampla, pois isso depende das espécies analisadas (Turner, 2005). 
A ecologia da paisagem estuda como os padrões espaciais influenciam os processos ecológicos.

Para isto, algumas teorias como a biogeografia de ilhas e metapopulações foram fundamentais, pois forneceram as sustentações teóricas a esse novo ramo da ciência, possibilitando oportunidades na construção e testes de hipóteses que relacionam padrões ambientais e processos ecológicos.

A teoria da biogeografia de ilhas proposta por MacArthur e Wilson sugere que a riqueza de espécies de uma ilha é determinada pelo balanço dinâmico entre as taxas de extinção e imigração, que por sua vez, são influenciadas pelas variáveis, tamanho e isolamento das ilhas. As taxas tendem a variar conforme o tamanho e isolamento da ilha/fragmento, e a riqueza de espécies de uma ilha é inversamente proporcional ao isolamento e diretamente proporcional ao seu tamanho. Quanto maior e mais próxima a ilha estiver do continente, maior será a variabilidade genética do local ou viceversa (Currie, 2010; Liira et al., 2014).

A teoria das metapopulações é um modelo proposto por Levins (1969). Inicialmente ela foi concebida como um programa de controle de pragas em áreas agrícolas, entretanto, vem sendo aplicada em estudos com espécies, em habitats naturais ou fragmentados. A teoria das metapopulações considera como objeto de estudo os fragmentos de habitats, diferentemente da teoria de MacArthur e Wilson que considera a ilha verdadeira.

O desenvolvimento da ecologia da paisagem se tornou viável com o avanço do sensoriamento remoto e dos sistemas de informações geográficas. Os diferentes sensores e resoluções (espacial, espectral, radiométrica e temporal) permitiram aos pesquisadores da ecologia da paisagem obterem dados, mais precisos e acurados, com rapidez e baixos custos (financeiros e temporais) de grandes porções da superfície da Terra, que é um subsídio essencial nos estudos de ecologia da paisagem. Deste modo, pode-se dizer que essas ciências são complementares.

No Brasil, as contribuições para o campo da ecologia da paisagem ainda são tímidas. As buscas no sistema Web of Science, até o ano de 2005, com as palavras-chave "ecologia + paisagem" em periódicos internacionais somaram apenas 30 resultados de autores brasileiros residentes no Brasil, sendo que mais de $50 \%$ desses consideraram a relação entre padrões espaciais e processos ecológicos, numa abordagem experimental-observacional, com uso de estatística.

A métrica da paisagem juntamente com a estatística foi o método mais utilizado no Brasil, considerando a produção de artigos em periódicos, dissertações e teses na última década (Metzger, 2007).

Em um país de dimensões continentais como o Brasil, o estudo da paisagem é particularmente importante pois possibilita, dentre outros fatores, obter informações a respeito da estrutura e das dinâmicas das alterações das paisagens, permitindo assim que se estabeleçam ações prioritárias para a conservação da biodiversidade e que se busque o desenvolvimento sustentável. Artigos de revisão são importantes para o desenvolvimento da ciência, pois geram um panorama sobre um assunto específico e permite que se definam prioridades de pesquisas futuras. Neste sentido, este artigo de revisão tem por objetivo discutir como o Sensoriamento Remoto tem sido utilizado na ecologia da paisagem nos últimos anos, considerando a temática, o tipo de abordagem e as técnicas mais utilizadas pelos pesquisadores em escala global.

\section{MATERIAIS E MÉTODOS}

O foco deste artigo está relacionado a três eixos norteadores: temática, abordagem e técnica. A partir daí, buscou-se respostas para algumas questões como: quais temas estão sendo mais pesquisados nos últimos anos? Que tipo de abordagem está sendo aplicada? E quais técnicas de Sensoriamento Remoto (SR) estão sendo utilizadas nas pesquisas de ecologia da paisagem?

As respostas para essas questões foram encontradas nos registros da literatura científica da base de dados Web of Science, considerando um fator de impacto maior que 2,0 (dois) e, linguagem preferencialmente em inglês, no intervalo temporal de 2010 a 2017. A aquisição das publicações foi realizada de forma sistemática utilizando como suporte o programa computacional StArt - State of the Art through systematic review (Zamboni et al., 2010), desenvolvido por pesquisadores da Universidade Federal de São Carlos (UFSCAR).

O programa computacional StArt atua como 
uma plataforma que auxilia o pesquisador na organização da pesquisa de forma sistemática. Ele possibilita que a organização da pesquisa seja realizada em três etapas: a) Planejamento. Etapa em que o pesquisador responde um protocolo sobre o assunto da pesquisa, esclarecendo o objetivo do trabalho, a questão central, as palavras-chave para busca e os critérios para definir quais artigos serão selecionados para pesquisa. b) $\mathrm{Na}$ segunda etapa denominada de execução, são inseridos no programa computacional o título, os autores, os periódicos e os resumos dos artigos selecionados em uma base de dados, que é armazenada digitalmente no formato bibtex.

É importante destacar que o programa computacional StArt não faz busca direta de artigos, pois o seu objetivo principal é o de organizar a pesquisa de forma sistemática.

Em seguida, foi realizada a leitura dos resumos, possibilitando a sua classificação em função da sua relevância por prioridade de leitura. Deste modo os artigos de maior relevância foram separados para posterior leitura mais aprofundada. c) Na terceira etapa foram obtidas informações estatísticas sobre o conjunto de artigos, tais como a porcentagem de artigos por tema, critério de seleção, periódico, semelhança, entre outras. A priori foram selecionados 237 artigos com base nas palavraschave "Physical Geography; Landscape; Transformation; Man; Impact; Anthropocene; Landscape Change; Human-Landscape Interactions; Human Impacts; Remote Sensing; Change Landscape". Desse total apenas 134 foram considerados relevantes para a pesquisa.

Os artigos selecionados foram separados em três categorias, sendo as duas iniciais elaboradas por Metzger (2007):

a) Em relação à temática:

- padrões espaciais: trabalhos baseados na descrição temática e estática (sem variação temporal) da paisagem ou de unidades da paisagem, descrição de impactos ambientais, caracterização de unidades de conservação ou áreas específicas, e elaboração de mapeamentos;

- dinâmica de uso e ocupação das terras: trabalhos voltados às causas, processos e consequências do uso e mudanças na cobertura das terras, baseados na comparação de padrões espaciais da paisagem em diferentes datas;

- planejamento ambiental: elaboração de zoneamentos ou medidas de manejo voltadas ao planejamento do meio físico, além de análise crítica de padrões de uso das terras e/ou soluções voltadas ao ordenamento territorial e ao melhor uso de recursos;

- percepção ambiental: trabalhos voltados à apreensão das opiniões de determinados setores da sociedade sobre algum aspecto da paisagem;

- metodologia/escala: descrição de avanços metodológicos; sugestão ou comparação de métodos para a análise de algum parâmetro da paisagem; análise de padrões e processos em diferentes escalas espaciais;

- relação entre padrões e processos: estudos que procuravam detectar e/ou compreender a influência de padrões espaciais da paisagem em processos ecológicos, ou vice-versa (ex.: efeitos da fragmentação na biodiversidade, efeitos de borda na ocorrência de espécies, entre outras); e

- outra: qualquer outro tema distinto dos anteriores.

b) Em relação à abordagem:

- observacional-descritiva: incluiu trabalhos descritivos e baseados unicamente na observação;

- observacional-experimental: delineamento do trabalho baseado em hipótese prévia, porém sem manipulação de parâmetros;

- experimental-manipulativa: delineamento do trabalho baseado em hipótese e com manipulação de parâmetros;

- modelagem: análise baseada em modelos (numéricos ou não) e simulação de situações;

- conceitual: apresentação e/ou discussão de conceitos;

- metodológica: enfoque na descrição ou avaliação de método proposto; e

- outra: qualquer outra abordagem que não as anteriores.

c) Em relação à técnica e sensores remotos:

- pré-processamento: trabalhos com processamento de dados brutos para calibração radiométrica da imagem, correção de distorções geométricas, remoção de ruído e realce, que visam melhorar a qualidade da imagem e classificação onde são atribuídas classes aos objetos presentes na imagem;

- radar/lidar: trabalhos com uso da tecnologia Light Detection And Ranging (LIDAR) ou Radar;

- VANT: trabalhos com uso de Veículos Aéreos Não Tripulados (VANT); e

- Outra: qualquer outra técnica distinta das anteriores.

Com base nos critérios estabelecidos anteriormente foi possível analisar de forma 
sistemática os avanços do Sensoriamento Remoto para a Ecologia da Paisagem, por meio da vasta literatura científica existente. A priori, notou-se que a maior contribuição do Sensoriamento Remoto para a Ecologia da Paisagem está na obtenção da informação de cobertura do solo.

\section{RESULTADOS E DISCUSSÃO}

A busca na literatura científica retornou 86 publicações de periódicos indexados a partir dos critérios adotados. Em relação aos temas trabalhados houve um equilíbrio entre padrão e dinâmica espacial, ambos com $24,4 \%$ do total. Planejamento e padrão/processo com $14 \%$ cada. Não foi encontrado nenhum artigo relacionado à percepção (Figura 1).

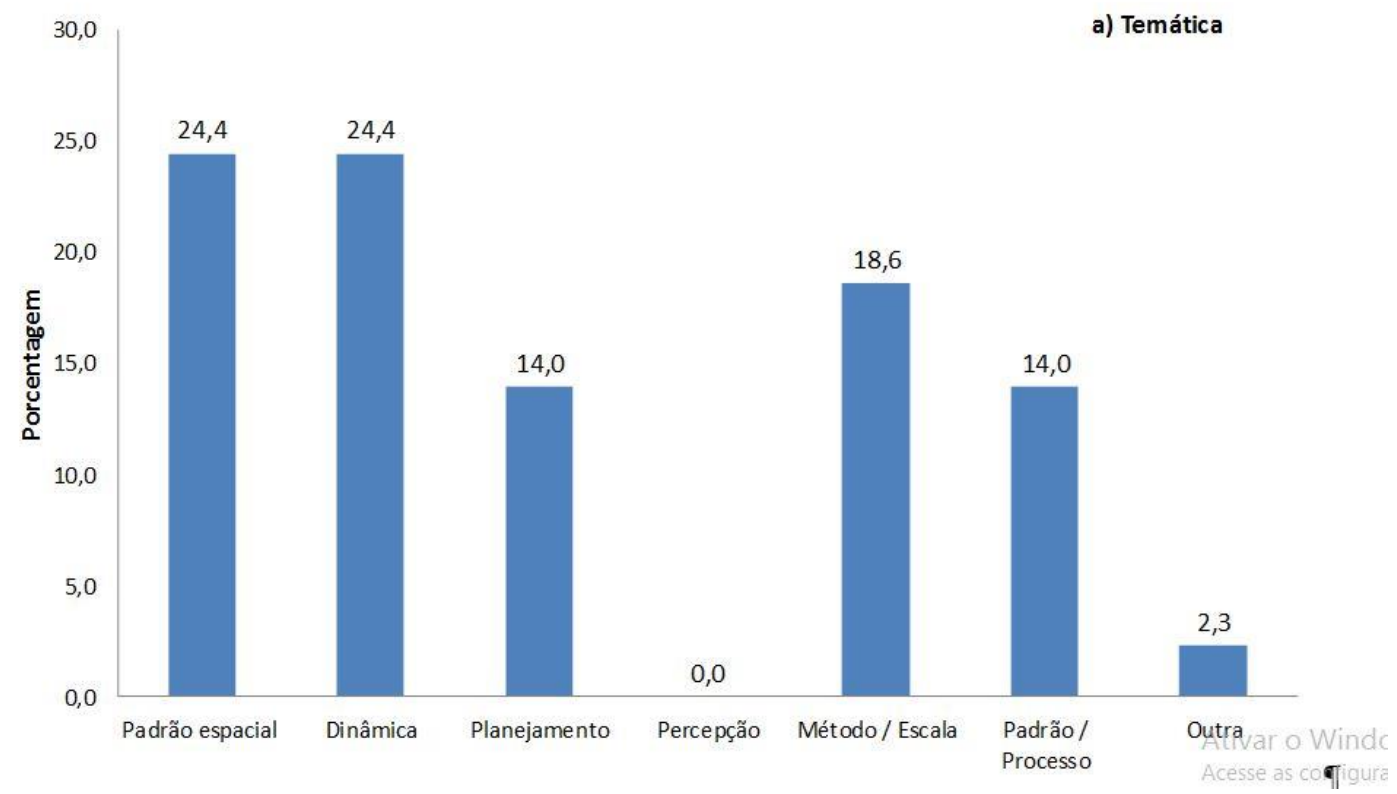

Figura 1 - Distribuição dos artigos em relação aos temas de pesquisa.

\section{Quais temáticas foram mais pesquisadas nos} últimos anos?

Geralmente os temas de pesquisa têm estreita relação com um estímulo pessoal do pesquisador, situação problema do momento, localização geográfica, dentre outras. Neste sentido, surge uma diversidade de trabalhos com os mais variados propósitos. No entanto, alguns temas como: padrão espacial, dinâmica e método/escala tiveram maior destaque.

A vegetação nativa fornece diversos serviços ecológicos à sociedade humana, tais como a retirada de carbono da atmosfera, proteção de rios e lagos, fornecimento de matéria prima, regulação térmica, viabilização da biodiversidade, entre outros. É fato que nos últimos anos as áreas ocupadas por vegetação nativa vêm sofrendo transformações drásticas por meio de manejos inadequados $\mathrm{e}$ as consequências são inevitáveis.

O desmatamento gera descontinuidades nos habitats cobertos por vegetação nativa e isto causa perturbações às espécies que dependem da integridade desses habitats, podendo levá-las a redução ou mesmo a extinção (Garbarino et al., 2012; Dahlin et al., 2014).

Estudos ecológicos necessitam de dados ambientais precisos e atualizados, tais como a caracterização da vegetação nativa, estrutura e organização da paisagem, ocupação e uso da terra, estrutura hidrográfica, entre outros. Esses estudos têm o objetivo de prever e explicar a distribuição espacial da biodiversidade.

Os recentes avanços em Sensoriamento Remoto têm facilitado a obtenção de dados espaciais e consequentemente a aplicação de análises espaciais quantitativas (métricas de paisagem) para os diferentes tipos de paisagens. As pesquisas em regiões tropicais têm demonstrado um grande interesse no entendimento dos padrões espaciais dos fragmentos de florestas, tendo como pilar a teoria da biogeografia de ilhas ou a teoria das metapopulações (Barrell \& Grant, 2013).

Em ambientes úmidos ou pantanosos o foco está direcionado à compreensão dos padrões das manchas de umidade utilizando imagens de alta resolução espacial (Arieira et al., 2011; Kelly et al., 2011). 
Os estudos relacionados à padrões espaciais geralmente procuram estabelecer um vínculo com os processos ecológicos, ou seja, a relação entre padrões e processos. Nesta linha de pesquisa os assuntos mais frequentes referem-se à estrutura biofísica da vegetação utilizando sensores ópticos na faixa espectral do visível em conjunto com imagens de radar (Betbeder et al., 2015), caracterização de ilhas de calor em paisagens urbanas com imagens termais (Xue et al., 2014; Zhang et al., 2013), além de estudos que relacionam a influência dos padrões de vegetação na fauna local (Narce et al., 2012; Simard et al., 2012).

Seguido dos temas padrão espacial e dinâmica, método/escala foi um dos temas mais pesquisados nos últimos anos. As pesquisas pertencentes a esta categoria visam principalmente a descrição, comparação de novos métodos ou validação de métodos já consagrados.

Existe na literatura científica uma preocupação com a acurácia dos dados obtidos a partir dos métodos de classificação, e também devido à escala espacial. Alguns pesquisadores questionam quais são as incertezas e qual seria o impacto nas análises de estrutura da paisagem. Os estudos sugerem precaução no uso de algoritmos de reamostragem para estudos de paisagem, sempre levando em consideração a estrutura original do dado, pois muitas vezes fragmentos de forma irregular não costumam manter a sua forma original após serem reamostrados (Oyana et al., 2014; Altamirano et al., 2012; Lechner et al., 2012; Vannier et al., 2011).

A escala é um assunto recorrente em ecologia da paisagem e muitos autores a consideram um elemento chave, que se não for corretamente considerado, pode comprometer a qualidade das análises. Neste sentido, alguns trabalhos buscam obter respostas para qual seria a escala ideal em cada tipo de análise. A abordagem multiescala tem sido muito utilizada para o monitoramento de problemas em habitats florestais.

O uso de imagens de satélite de variadas resoluções espaciais, juntamente com outros produtos como o Light Detection And Ranging (LIDAR) tem contribuído para o processo de tomada de decisão e consequentemente para a gestão sustentável (Osberger et al., 2014; Vannier et al., 2011).
Obviamente que diversos outros temas foram encontrados na busca, como: modelagem geomorfológica de paisagens marinhas usando sensores ópticos (Hamylton e Spencer, 2011); estudos de estrutura e função de rios utilizando imagens de radar, fotos aéreas e imagens de alta resolução (Carbonneau et al., 2012); monitoramento de serviços ecossistêmicos (Fegraus et al., 2012); modelagem ecológica (Hamylton et al., 2012; Sardà-Palomera et al., 2012), dentre outros. No entanto, o objetivo deste trabalho foi fornecer um panorama geral dos temas com maior frequência nos últimos anos.

\section{Quais abordagens estão sendo adotadas} atualmente na ecologia da paisagem?

A abordagem metodológica teve uma porcentagem de artigos bem superior que as outras abordagens, com aproximadamente $61 \%$ dos artigos pesquisados, seguida da categoria de modelagem com $17,4 \%$. Grande parte dos artigos analisados abordaram os temas de ecologia da paisagem de forma avaliativa (Buma, 2012) ou por aplicação de métodos recentes, como uso de sensores hiperespectrais para avaliar o estado de conservação da paisagem e uso de sensores a laser para verificar a conectividade da paisagem em três dimensões (Casalegno et al., 2017; Schmidt et al., 2017), ou já consagrados como os estudos temporais a partir de imagens do satélite Landsat (Xue Xing-Hua \& Yuan Long-Yi, 2013; Du et al., 2013; Kim \& Daigle, 2011). Uma porcentagem baixa de artigos $(3,5 \%)$ abordaram os temas com foco puramente conceitual. $\mathrm{O}$ tema modelagem teve a segunda maior contribuição, com foco direcionado para a modelagem ecológica (Figura 2).

A avaliação de métodos tem um enfoque na qualidade dos resultados gerados em análises de ecologia da paisagem, que geralmente são afetadas por (i) incertezas na classificação do tema, (ii) escala espacial e (iii) erro de classificação. Alguns autores (Lechner et al., 2012) questionam se existe por parte dos ecologistas de paisagem uma preocupação ao conduzir as suas análises.

A conclusão é que muitas vezes os ecologistas de paisagem lidam com os produtos finais como mapas de cobertura do solo e não estão familiarizados com os métodos computacionais para criá-los, e isto pode se tornar um problema para a descoberta de relações existentes ou espúrias (Turner et al., 2001). 


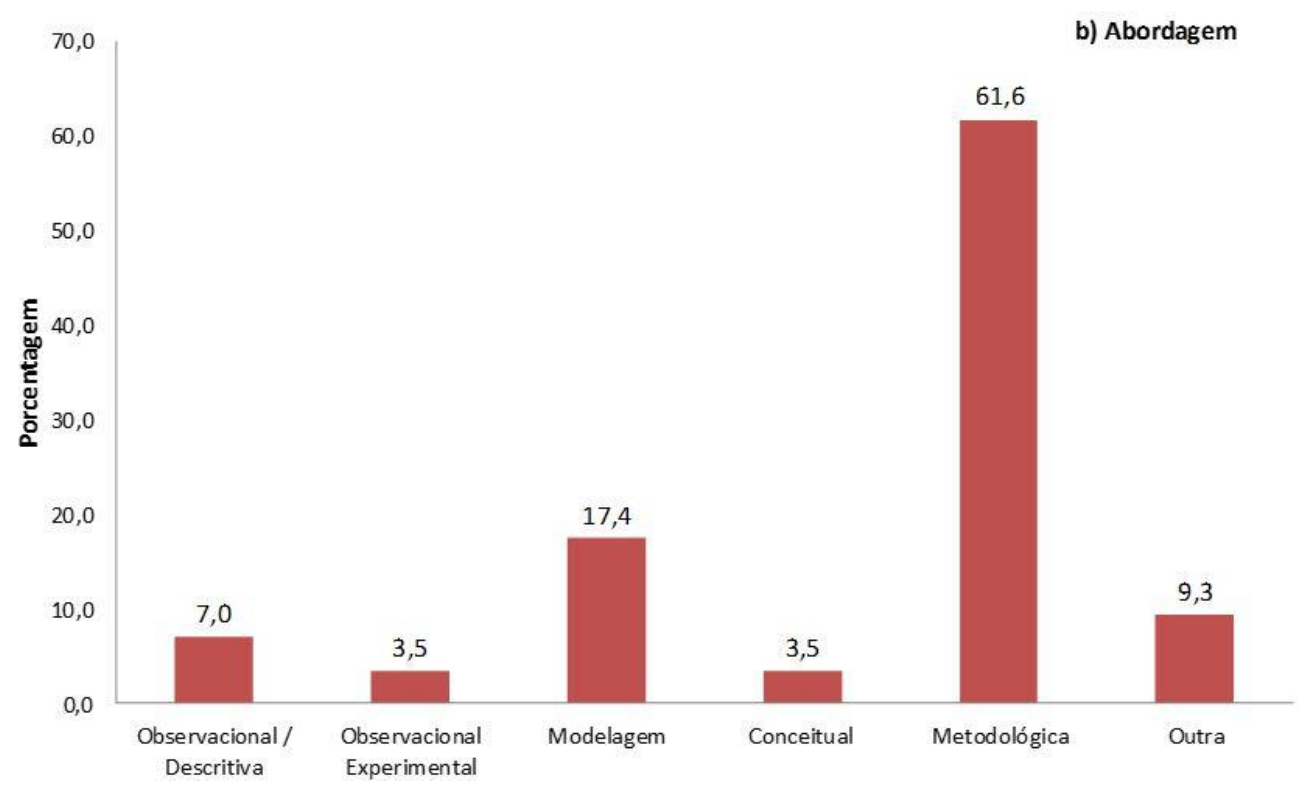

Figura 2 - Distribuição dos artigos em relação à abordagem dos temas de pesquisa.

Uma característica da abordagem conceitual é a aplicação ou discussão de teorias já estabelecidas ou novas. São trabalhos bem elaborados e com hipóteses muito bem delineadas.

Talvez seja essa a explicação para o baixo percentual encontrado, considerando o grau de dificuldade e maturidade para a construção de um artigo neste tipo de abordagem. Alguns dos trabalhos encontrados nesta categoria discutem o potencial do sensoriamento remoto na estimativa de biodiversidade utilizando índices e o conceito de paisagem ribeirinha "Riverscape" que utiliza informações sobre a distribuição espacial dos habitats em todo o sistema fluvial (Carbonneau et al., 2012; Rocchini et al., 2014).

Existe um consenso na literatura em relação à importância dos dados e técnicas de sensoriamento remoto principalmente para modelagem de paisagens. Nesta abordagem o objetivo principal é fornecer subsídio aos tomadores de decisão para indicar áreas prioritárias de conservação por meio de simulações de cenários, onde é possível acompanhar espacialmente e temporalmente a fragmentação de um habitat (Carolina et al., 2014).

A modelagem ecológica têm se utilizado do sensoriamento remoto nos mais variados ecossistemas. No ambiente marinho os modelos estudam o comportamento de algas e corais mortos e vivos, sem a compreensão da distribuição espacial de cobertura dos corais e os fatores que geram influência na escala de paisagem não é possível gerir de forma eficiente as reservas marinhas destes ecossistemas
(Hamylton, 2012; Hamylton et al., 2012).

Além dos estudos direcionados à flora marinha, foram encontrados trabalhos com foco na geomorfologia costeira, onde existe uma preocupação em investigar os processos que influenciam a alternância de areia na costa marinha utilizando mapas de habitat derivados a partir de imagens de sensoriamento remoto (Hamylton \& Spencer, 2011).

Outros modelos (Cleckner et al., 2011) visam o estudo da proliferação de insetos que podem gerar um risco de saúde para população, tendo em vista que estes carregam consigo patógenos causadores de doenças.

Os autores destacam a importância dos dados de sensoriamento remoto na obtenção da distribuição de habitats na paisagem. De fato, os modelos têm sido aplicados em diversas situações problemas buscando um maior entendimento dos processos e dinâmicas ambientais, entretanto, a modelagem de mudança de uso ou cobertura do solo (Kumar et al., 2013; Gusmão et al., 2014) se destaca pelo fato de se tornar uma informação base não só para os modelos, mas para uma diversidade de estudos.

Quais técnicas de sensoriamento remoto têm sido utilizadas em conjunto com a ecologia da paisagem?

O conjunto de técnicas empregadas nos estudos de ecologia da paisagem abrange uma vasta gama de dados, algoritmos, procedimentos, que se mal empregados podem gerar resultados não muito coerentes. No sensoriamento remoto ao utilizar um dado bruto, oriundo de sensor ótico 
acoplado em plataforma orbital, existe a necessidade de manipulá-lo para que o mesmo se torne adequado para as análises.

Esse procedimento é feito por meio de técnicas de pré-processamento como o realce da imagem, calibração radiométrica, correção das distorções geométricas, remoção de ruídos, entre outras.

De posse do dado pré-processado, realiza-se a classificação das imagens, onde os elementos da

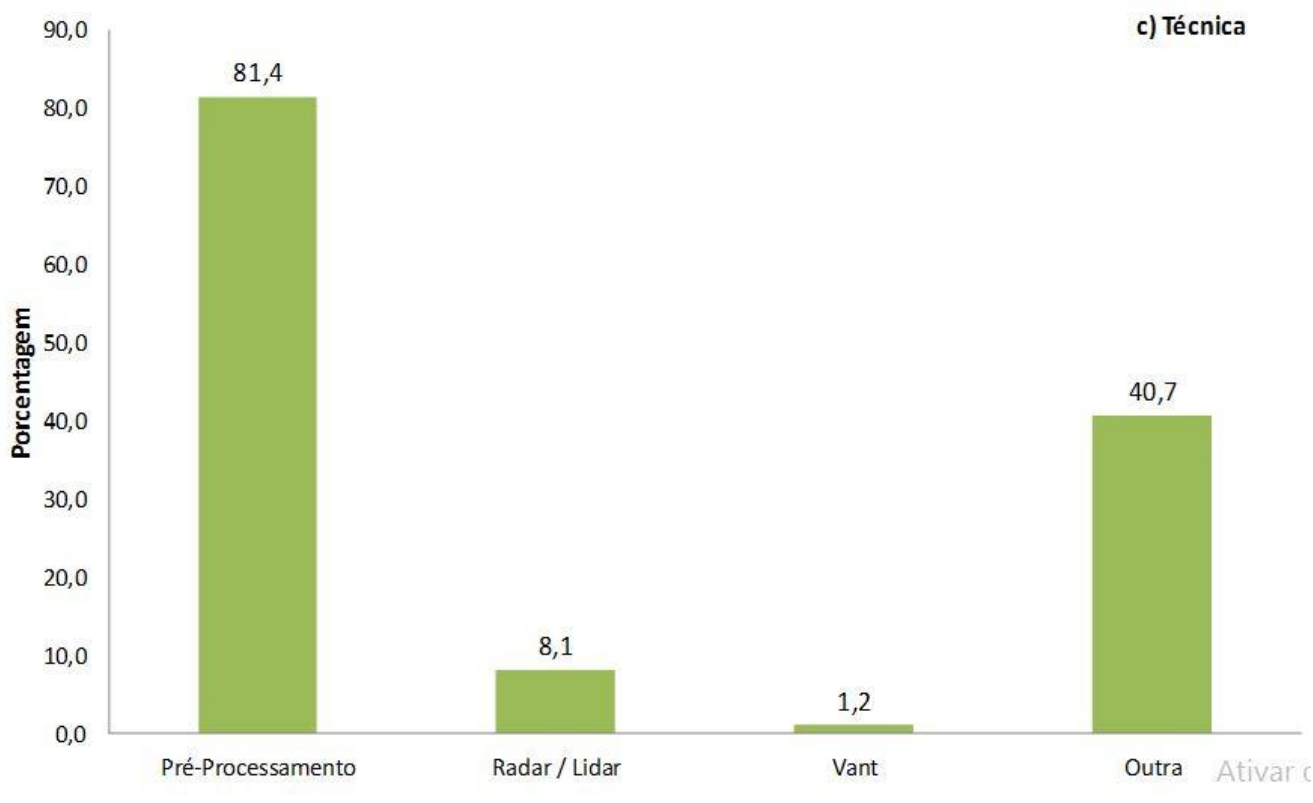

Figura 3 - Distribuição dos artigos em relação às técnicas utilizadas nas pesquisas.

A figura 4 mostra certo equilíbrio entre as linhas de pesquisas encontradas na categoria préprocessamento. $\mathrm{O}$ mapeamento de uso $\mathrm{e}$ cobertura do solo foi a linha de pesquisa de maior interesse com $23 \%$ do total, um valor não muito distante de estudos com foco na dinâmica temporal, com $20 \%$.

Este resultado pode ser considerado coerente, uma vez que os estudos em ecologia da paisagem consideram os padrões espaciais que podem ser extraídos de mapas de uso e cobertura do solo e o fator temporal, que tornam possíveis as análises de influência dos padrões espaciais nos processos ecológicos.

Para o mapeamento de uso e cobertura do solo os pesquisadores têm utilizado imagens de resolução espacial média (Chawla et al., 2012; Yang et al., 2012; Marston et al., 2014) e alta (Ozdemir et al., 2012; Recio et al., 2014; Kane et al., 2014; Gusmão et al., 2014), assim como a vetorização de mapas (Donnelly, 2011) e até mesmo a criação de programas específicos (Körting et al., 2013) para atender as suas demandas.

A maioria dos trabalhos com foco direcionado imagem (pixels) são agrupados em classes distintas.

A maior parte dos artigos analisados $(81,4 \%)$ utilizaram as técnicas de pré-processamento, em seguida com $40,7 \%$ está o uso de outras técnicas específicas para o processamento de dados oriundos de Radar/LIDAR ou VANT, sendo que estas duas últimas juntas não somaram $10 \%$ do total (Figura 3). à classificação utilizou métodos clássicos como a classificação supervisionada ou não supervisionada, baseada em pixel ou a técnica de classificação híbrida que utiliza a interpretação visual e o índice de vegetação NDVI para reduzir o efeito de sombreamento no resultado.

Segundo os autores o algoritmo de classificação confunde os pixels sombreados com outras classes de resposta espectral semelhante, sendo que geralmente essa confusão ocorre entre as classes de sombra e vegetação, neste sentido, ao utilizar o NDVI o problema pode ser amenizado (Reddy et al., 2013; Garbarino et al., 2012; Altamirano et al., 2012).

A classificação sub-pixel, ou classificação por redes neurais foi observada em trabalhos atuais, em que algoritmos são treinados para aprender conforme são alimentados com novos padrões e assim minimizam a probabilidade de erros na predição (Christopher et al., 2014).

Os ecologistas de paisagens enfrentam problemas ao classificar algumas paisagens devido a sua heterogeneidade e diferentes escalas. Ao reduzir a escala, os fragmentos de paisagem menores nem sempre mantém a sua 
forma original e isto pode gerar problemas na extração de métricas da paisagem. As pesquisas sugerem que é possível reduzir a escala, ou seja, degradar o pixel, e ao mesmo tempo manter a precisão da classificação utilizando a técnica de sub-pixel, no entanto o nível de precisão depende da heterogeneidade da paisagem (Frazier, 2014; Frazier \& Wang, 2013; Valentini et al., 2014).

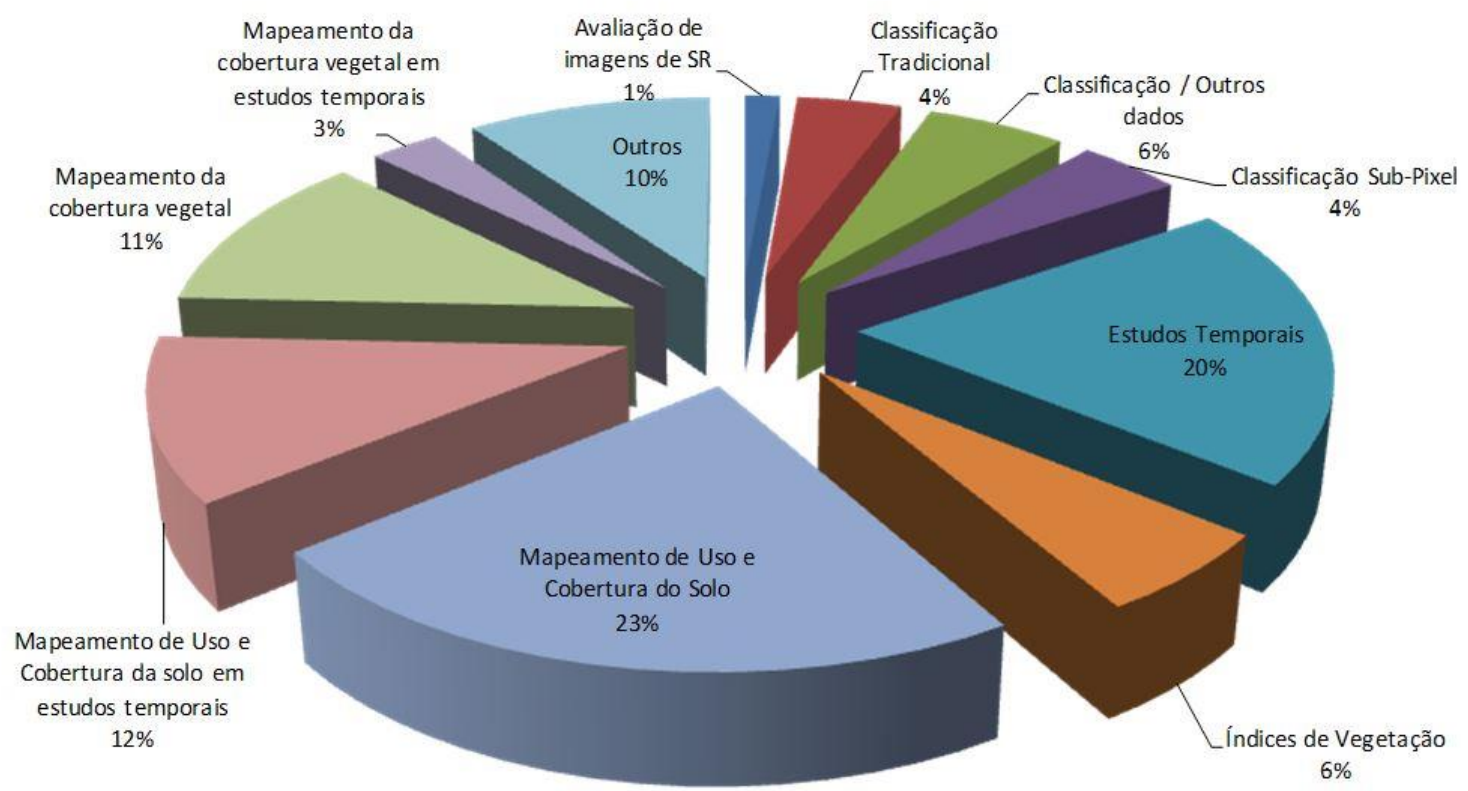

Figura 4 - Estudos verificados na técnica de pré-processamento.

Os estudos atuais que levam em consideração o fator tempo demonstraram um interesse maior na detecção de alterações na paisagem ao longo do tempo. As pesquisas se distribuem nas mais diversas áreas como turismo (Gkoltsiou et al., 2013), monitoramento da vegetação (Franks et al., 2013; Godar et al., 2012), estudos urbanos (Feng \& Li, 2012; Li \& Yin, 2013; Li et al., 2012), sistema aquático (Tulbure et al., 2013) e regiões desérticas (Hirche et al., 2011). Existe um consenso entre estes autores da importância do sensoriamento remoto para as análises espaço-temporais. O dado utilizado em cada estudo depende da sua particularidade e objetivo, entretanto para esta linha de pesquisa foi observado a predominância das imagens do satélite Landsat seguida pelo uso das imagens MODIS. É muito provável que a preferência por estes produtos seja pelo fato da disponibilidade de acesso e ampla cobertura temporal.

Nos mapeamentos de uso e cobertura do solo foi observada uma tendência no uso de imagens de alta resolução espacial, além de outros produtos como os oriundos de sensores LIDAR, que fornecem a estrutura tridimensional da paisagem. A resolução espacial nos estudos em ecologia da paisagem é um fator importante, alguns autores (Oyana et al., 2014) observaram que os mapeamentos com imagens de satélite, com resolução espacial de 30 metros ou mais detalhada são melhores para análises da dinâmica da paisagem, e isto corrobora com a preferência observada pelo uso de imagens Landsat quando não há possibilidade do uso de imagens de alta resolução, pois o custo para aquisição destas ainda é elevado.

De modo geral, o grande avanço do sensoriamento remoto na ecologia de paisagens está na evolução computacional que permitiu análises mais acuradas com maior agilidade e precisão. A geração de novos programas e a possibilidade de adaptação dos mesmos é um grande avanço para a área além dos diversos produtos de sensores remotos disponíveis atualmente como imagens de alta resolução espacial que chegam a ter um tamanho de pixel na escala centimétrica, o que requer programas computacionais mais robustos para as análises, no entanto oferece resultados mais detalhados.

As técnicas de processamento de imagens digitais oriundas de sensores remotos estão em constante evolução, como no caso das redes neurais e as técnicas de machine learning e deep learning, que apesar de não muito utilizadas na ecologia da paisagem devido a sua complexidade, garantem aos seus usuários uma saída para um grande problema do sensoriamento remoto que é a mistura espectral de alvos. 


\section{CONCLUSÕES}

O Sensoriamento Remoto têm sido essencial nos estudos de ecologia da paisagem, pois, sem as tecnologias desenvolvidas, algumas análises de grandes porções da superfície da Terra, tais como os biomas brasileiros, seriam inviáveis. A sua maior contribuição está na flexibilidade de uso em diversas linhas de pesquisa, o que o torna uma ferramenta complementar não só da ecologia da paisagem, mas de várias ciências.

Atualmente, os temas de maior interesse estão relacionados à dinâmica e padrão espacial da paisagem, isto reflete o momento ambiental pelo qual o planeta está passando. Com a degradação dos recursos naturais e as mudanças climáticas globais, é compreensível que os interesses científicos se voltem para essas questões. O entendimento do comportamento do padrão espacial da paisagem ao longo do tempo fornece subsídio, no Brasil, para a gestão ambiental mais eficiente, pois os instrumentos de planejamento, comando e controle, e econômicos necessitam de informações oriundas da análise de paisagem.

Os resultados da busca bibliográfica demonstraram que os pesquisadores têm abordado os temas de pesquisa em sua maioria de forma metodológica, ou seja, uma abordagem onde predominam os métodos e técnicas. Houve uma porcentagem muita baixa de estudos com propósito puramente conceitual e, seria oportuno se esta linha de pesquisa se desenvolvesse, para, a partir daí, formular hipóteses mais consistentes com uma base conceitual mais atualizada.

As técnicas tradicionais de classificação de imagens, que é uma das etapas do préprocessamento, ainda é uma das mais aplicadas atualmente com suporte de índices de vegetação e mapeamento a LASER nos estudos de ecologia da paisagem. Foi identificado apenas um estudo com uso de Veículo Aéreo não Tripulado VANT (Rakan et al., 2015), que representa uma tecnologia relativamente nova e promete trazer resultados satisfatórios para a comunidade que utiliza as informações de mapeamento de cobertura do solo. Uma vez que os VANTs podem realizar mapeamentos com câmeras de alta definição ou mesmo multiespectrais, além de mapeamento a laser, o que faz com que o uso desta ferramenta tenha o seu potencial expandido.

Em nenhum dos artigos analisados foi identificado o uso de uma ferramenta atual idealizada pela empresa Google denominada Google Earth Engine (https://earthengine. google.org/\#intro). Essa ferramenta possui um banco de dados de produtos satelitários com observações de mais de 40 anos, os produtos estão disponíveis em uma única plataforma onde o usuário por meio de linguagem de programação javascript pode realizar análises de detecção de mudanças, quantificação de diferenças, mapeamento da cobertura da terra, estimativa de biomassa, carbono dentre outros, tudo isso em escala geográfica global em um período de tempo muito menor se comparado aos programas computacionais tradicionais de processamento de imagens satelitárias.

Com os desafios ambientais da atualidade, devido à perda de biodiversidade, qualidade e quantidade de recursos hídricos, expansão urbana e de atividades agropecuárias sem controle, além das mudanças climáticas globais, a produção científica a respeito da análise da paisagem tende a crescer, tanto em nível global quanto a nível nacional. Esse crescimento será viabilizado pela disponibilidade de dados de Sensoriamento Remoto, tanto por computação em nuvem, como é o caso do Google Earth Engine, quanto por sensores acoplados em veículos aéreos não tripulados.

Tratando-se de análises em ecologia da paisagem que geralmente trabalha com escala macro, esta seria uma alternativa muito eficaz para análises que envolvam a superfície terrestre.

\section{AGRADECIMENTOS}

À Coordenação de Aperfeiçoamento de Pessoal de Nível Superior (CAPES), pela concessão de bolsa de Doutorado ao primeiro autor.

\section{REFERÊNCIAS}

ALTAMIRANO, A.; MIRANDA, A.; JIMÉNEZ, C Incertidumbre de los índices de paisaje en el análisis de la estructura espacial. Bosque (Valdivia), v. 33, n. 2, p. 13-14, 2012.

ARIEIRA, J.; KARSSENBERG, D.; JONG, S.M.; ADDINK, E.A.; COUTO, E.G.; CUNHA, C.N.; SKØIEN, J.O. Integrating field sampling, geostatistics and remote sensing to map wetland vegetation in the Pantanal, Brazil. Biogeosciences, v. 8, n. 3, p. 667-686, 2011.

BARRELL, J. \& GRANT, J. Detecting hot and cold spots in a seagrass landscape using local indicators of spatial association. Landscape Ecology, v. 28, n. 10, p. 2005-2018, 2013. 
BETBEDER, J.; HUBERT-MOY, L.; BUREL, F.; CORGNE, S.; BAUDRY, J. Assessing ecological habitat structure from local to landscape scales using synthetic aperture radar. Ecological Indicators, v. 52, p. 545-557, 2015. Disponível em: <http://dx.doi.org/10.1016/j.ecolind.2014.11.009>. .

BUMA, B. Evaluating the utility and seasonality of NDVI values for assessing post-disturbance recovery in a subalpine forest. Environmental Monitoring and Assessment, v. 184, n. 6, p. 3849-3860, 2012.

CARBONNEAU, P.; FONSTAD, M.A.; MARCUS, W.A.; DUGDALE, S.J. Making riverscapes real. Geomorphology, v. 137, n. 1, p. 74-86, 2012. Elsevier B.V. Disponível em: <http://dx.doi.org/10.1016/j.geomorph.2010.09.030>.

CAROLINA, G.S.; LISIANE, Z.; ROSÂNGELA, A.T.B.; LUIS, M.T.C.; HELENA, M.R.A.; MARGARETE, M.L.V. Análise da Fragmentação Florestal da Área de Proteção Ambiental Coqueiral, COQUEIRAL, p. 631-644, 2014.

CHAWLA, A.; KUMAR, A.; LAL, B.; SINGH, R.D.; THUKRAL, A.K. Ecological Characterization of High Altitude Himalayan Landscapes in the Upper Satluj River Watershed, Kinnaur, Himachal Pradesh, India. Journal of the Indian Society of Remote Sensing, v. 40, n. 3, p. 519-539, 2012.

CHRISTOPHER, G.M.F.; MARK, D.; RICHARD, P. A.; PATRICK, G.; DAVID, R.J.P.; QIAN, W.; JIAMIN, Q.; PHILIP, S.C. A random forest approach for predicting the presence of Echinococcus multilocularis intermediate host Ochotona spp. presence in relation to landscape characteristics in western China. Applied Geography, v. 55, p. 176-183, 2014. Elsevier Ltd. Disp. em: <http://linkinghub.elsevier.com/ retrieve/pii/S0143622814002021>.

CLECKNER, H.L.; ALLEN, T.R.; SCOTT, B.A. Remote sensing and modeling of mosquito abundance and habitats in Coastal Virginia, USA. Remote Sensing, v. 3, n. 12, p. 2663-2681, 2011.

CURRIE, D.J. The Theory of Island Biogeography Revisited. BioScience, v. 60, n. 11, p. 952-953, 2010.

DAHLIN, K.M.; ASNER, G.P.; FIELD, C.B. Linking vegetation patterns to environmental gradients and human impacts in a mediterranean-type island ecosystem. Landscape Ecology, p. 1571-1585, 2014.

DONNELLY, S. Land-use portfolios and the management of private landholdings in south-central Indiana. Regional Environmental Change, v. 11, n. 1, p. 97-109, 2011.

DU, P.; XIA, J.; DU, Q.; LUO, Y.; TAN, K. Evaluation of the spatio-temporal pattern of urban ecological security using remote sensing and GIS. International Journal of Remote Sensing, v. 34, n. 3, p. 848-863, 2013.

FEGRAUS, E.H.; ZASLAVSKY, I.; WHITENACK, T.; JAN DEMPEWOLF, J.A.; AHUMADA, K.L.; SANDY, J.A. Interdisciplinary decision support dashboard: A new framework for a tanzanian agricultural and ecosystem service monitoring system pilot. IEEE Journal of Selected Topics in Applied Earth Observations and Remote Sensing, v. 5, n. 6, p. 1700-1708, 2012.

FENG, L. \& LI, H. Spatial Pattern Analysis of Urban Sprawl : Case Study, n. September, p. 263-269, 2012.

FRANKS, S.; MASEK, J.G.; TURNER, M.G. Monitoring forest regrowth following large scale fire using satellite data: A case study of Yellowstone National Park, USA. European Journal of Remote Sensing, v. 46, n. 1, p. 561-569, 2013.

FRAZIER, A.E. A new data aggregation technique to improve landscape metric downscaling. Landscape Ecology, v. 29, n. 7, p. 1261-1276, 2014.

FRAZIER, A.E. \& WANG, L. Modeling landscape structure response across a gradient of land cover intensity. Landscape Ecology, v. 28, n. 2, p. 233-246, 2013.

GARBARINO, M.; MONDINO, E.B.; LINGUA, E.; NAGEL, T.A.; DUKIĆ, V.; GOVEDAR, Z.; MOTTA, R. Gap disturbances and regeneration patterns in a Bosnian old-growth forest: A multispectral remote sensing and ground-based approach. Annals of Forest Science, v. 69, n. 5, p. 617-625, 2012.

GKOLTSIOU, A.; TERKENLI, T.S.; KOUKOULAS, S. Landscape indicators for the evaluation of tourist landscape structure. International Journal of Sustainable Development \& World Ecology, v. 20, n. 5, p. 461-475, 2013. Disp. em: <http://www.tandfonline.com.esc-web.lib.cbs.dk/doi/full/10. 1080/13504509.2013.827594\#.U7P5fRYWnbQ>.

GODAR, J.; TIZADO, E.J.; POKORNY, B. Who is responsible for deforestation in the Amazon? A spatially explicit analysis along the Transamazon Highway in Brazil. Forest Ecology and Management, v. 267, p. 58-73, 2012. Elsevier B.V. Disp. em: <http://dx.doi.org/10.1016/j.foreco.2011.11.046>.

HAMYLTON, S. A comparison of spatially explicit and classic regression modelling of live coral cover using hyperspectral remote-sensing data in the Al Wajh lagoon, Red Sea. International Journal of Geographical Information Science, v. 26, n. 11, p. 1-15, 2012.

HAMYLTON, S.M. \& SPENCER, T. Geomorphological modelling of tropical marine landscapes: Optical remote sensing, patches and spatial statistics. Continental Shelf Research, v. 31, n. 2 SUPPL., p. 151-161, 2011.

HAMYLTON, S.; SPENCER, T.; HAGAN, A. Spatial modelling of benthic cover using remote sensing data in the Aldabra lagoon, western Indian Ocean. Marine Ecology Progress Series, v. 460, p. 35-47, 2012.

HIRCHE, A.; SALAMANI, M.; ABDELLAOUI, A.; BENHOUHOU, S.; VALDERRAMA, J.M. Landscape changes of desertification in arid areas: The case of south-west Algeria. Environmental Monitoring and Assessment, v. 179, n. 1-4, p. 403-420, 2011

KANE, K.; CONNORS, J.P.; GALLETTI, C.S. Beyond fragmentation at the fringe: A path-dependent, high-resolution analysis of urban land cover in Phoenix, Arizona. Applied Geography, v. 52, p. 123-134, 2014. Elsevier Ltd. Disp. em: <http://dx.doi.org/10.1016/j.apgeog.2014.05.002>. .

KELLY, M.; TUXEN, K.A.; STRALBERG, D. Mapping changes to vegetation pattern in a restoring wetland: Finding pattern metrics that are consistent across spatial scale and time. Ecological Indicators, v. 11, n. 2, p. 263-273, 2011. Elsevier Ltd. Disp. em: <http://dx.doi.org/10.1016/j.ecolind.2010. 05.003>. .

KIM, M.K. \& DAIGLE, J.J. Detecting vegetation cover change on the summit of Cadillac Mountain using multi-temporal remote sensing datasets: 1979, 2001, and 2007. Environmental Monitoring and Assessment, v. 180, n. 1-4, p. 63-75, 2011.

KÖRTING, T.S.; GARCIA FONSECA, L.M.; CÂMARA, G. GeoDMA-Geographic Data Mining Analyst. Computers and Geosciences, v. 57, p. 133-145, 2013. Elsevier. Disp. em: <http://dx.doi.org/10.1016/j.cageo.2013.02.007>.

KUMAR, S.; RADHAKRISHNAN, N.; MATHEW, S. Land use change modelling using a Markov model and remote sensing. Geomatics, Natural Hazards and Risk, v. 5, n. 2, p. 145-156, 2013. Disp. em: <http://www.tandfonline.com/doi/abs/10. 1080/19475705.2013.795502>.

LECHNER, A.M.; LANGFORD, W.T.; BEKESSY, S.A.; JONES, S. D. Are landscape ecologists addressing uncertainty in their remote sensing data? Landscape Ecology, v. 27, n. 9, p. 1249-1261, 2012.

LEVINS, R. Some Demographic and Genetic Consequences of Environmental Heterogeneity for Biological Control. Bulletin of the Entomological Society of America, p. 15:237-240, 1969.

LI, C.F. \& YIN, J.Y. A Study on Urban Thermal Field of Shanghai Using Multi-source Remote Sensing Data. Journal of the Indian Society of Remote Sensing, v. 41, n. 4, p. 1009 1019, 2013.

LI, X.; ZHOU, W.; OUYANG, Z.; XU, W.; ZHENG, H. Spatial pattern of greenspace affects land surface temperature: Evidence from the heavily urbanized Beijing metropolitan area, 
China. Landscape Ecology, v. 27, n. 6, p. 887-898, 2012.

LIIRA, J.; JÜRJENDAL, I.; PAAL, J. Do forest plants conform to the theory of island biogeography: The case study of bog islands. Biodiversity and Conservation, v. 23, n. 4, p. 10191039, 2014.

METZGER, J.P. O que é ecologia de paisagens. Biota Neotropica, v. 1, p. 1-9, 2001. Disp. em: <http://www.biotaneo tropica.org.br/v1n12/pt/abstract?thematicreview+BN00701122001>.

METZGER, J.P. Diagnóstico da pesquisa em ecologia de paisagens no Brasil (2000-2005). Biotaneotropica, v. 7, n. 3, p. 21-29, 2007.

NARCE, M.; MELONI, R.; BEROUD, T.; PLÉNEY, A; RICCI, J.C. Landscape ecology and wild rabbit (Oryctolagus cuniculus) habitat modeling in the Mediterranean region., v. 2, p. 277-284, 2012.

OSBERGER, A.; STRASSER, T.; RIEDLER, B.; ADAMCZYK, J.; LANG, S.; PERNKOPF, L. Multi-scale Forest Habitat Monitoring Using Remote Sensing Data, n. 2014, p. 49-56, 2014.

OYANA, T.J.; JOHNSON, S.J.; WANG, G. Landscape metrics and change analysis of a national wildlife refuge at different spatial resolutions. International Journal of Remote Sensing, v. 35, n. 9, p. 3109-3134, 2014. Disp. em: <http://www. tandfonline.com/doi/abs/10.1080/01431161.2014.903443>. .

OZDEMIR, I.; MERT, A.; SENTURK, O. Predicting Landscape Structural Metrics Using Aster Satellite Data. Journal of Environmental Engineering and Landscape Management, v. 20 , n. 2, p. 168-176, 2012.

RAKAN, A.; ZAHAWI, J. P.; DANDOIS, K. D.; HOLL, D.; NADWODNY, J.L. \& REID, E.C.E. Using lightweight unmanned aerial vehicles to monitor tropical forest recovery. Biological Conservation, v. 186, p. 287-295, 2015. Elsevier Ltd. Disp. em: <http://linkinghub.elsevier.com/retrieve/ pii/S0006320715001421>.

RECIO, M.R.; MATHIEU, R.; VIRGÓS, E.; SEDDON, P.J. Quantifying fine-scale resource selection by introduced feral cats to complement management decision-making in ecologically sensitive areas. Biological Invasions, v. 16, n. 9, p. 1915-1927, 2014

REDDY, C.S.; JHA, C.S.; DADHWAL, V.K. Assessment and monitoring of long-term forest cover changes in Odisha, India using remote sensing and GIS. Environmental Monitoring and Assessment, v. 185, n. 5, p. 4399-4415, 2013.

ROCCHINI, D.; HERNÁNDEZ-STEFANONI, J.L.; HE, K.S. Advancing species diversity estimate by remotely sensed proxies: a conceptual review. Ecological Informatics, v. 25, p. 22-28, 2014. Elsevier B.V. Disp. em: <http://dx. doi.org/10.1016/j.ecoinf.2014.10.006>. .

SARDÀ-PALOMERA, F.; PUIGCERVER, M.; BROTONS, L.; DOMINGO, J. Modelling seasonal changes in the distribution of Common Quail Coturnix coturnix in farmland landscapes using remote sensing. , p. 703-713, 2012.

SCHMIDT, J.; FASSNACHT, F.E.; LAUSCH, A.; SCHMIDTLEIN, S. Assessing the functional signature of heathland landscapes via hyperspectral remote sensing. Ecological Indicators, v. 73, p. 505-512, 2017.

SIMARD, M.; POWELL, E. N.; RAFFA, K. F.; TURNER, M. G. What explains landscape patterns of tree mortality caused by bark beetle outbreaks in Greater Yellowstone? Global Ecology and Biogeography, v. 21, n. 5, p. 556-567, 2012.
STEFANO, C.; KAREN, A.; DANIEL, T.C.C.; STEVEN, H.; KEVIN, J.G.; Ecological connectivity in the three-dimensional urban green volume using waveform airborne lidar. Scientific Reports, v. 7, 2017.

TROLL, C. Landscape ecology (geo-ecology) and biogeocenology: a terminological study. Geoforum, v. 8, p. 43-46, 1971.

TULBURE, M.G.; KININMONTH, S.J.; BROICH, M. Spatiotemporal dynamics of surface water networks across a global biodiversity hotspot. Modsim, v. 114012, p. 70, 2013. IOP Publishing.

TURNER, M.G. Landscape Ecology: What Is the State of the Science? Annual Review of Ecology, Evolution, and Systematics, v. 36, n. 1, p. 319-344, 2005.

TURNER, M.G.; GARDNER, R.H.; O'NEILL, R.V. Landscape Ecology in theory and practice, 2001.

VALENTINI, E.; TARAMELLI, A.; FILIPPONI, F.; GIULIO, S. An effective procedure for EUNIS and Natura 2000 habitat type mapping in estuarine ecosystems integrating ecological knowledge and remote sensing analysis. Ocean and Coastal Management, 2014. Elsevier Ltd. Disp. em: <http://dx.doi.org/10.1016/j.ocecoaman.2014.07.015>. .

VANNIER, C.; VASSEUR, C.; HUBERT-MOY, L.; BAUDRY, J. Multiscale ecological assessment of remote sensing images. Landscape Ecology, v. 26, n. 8, p. 1053-1069, 2011.

XUE, XING-HUA; YUAN, LONG-YI. Study on the Fluvial Landscape Patterns of Upper Jingjiang Segment, Yangtze River, Using Multi-temporal Remote-Sensing Imageries. 2013 Fifth International Conference on Measuring Technology and Mechatronics Automation, p. 1095-1102, 2013. Disp. em: <http://ieeexplore.ieee.org/lpdocs/epic03/wrapper.htm?ar number $=6493921>$.

XUE, Y.; FUNG, T.; TSOU, J. Urban thermal landscape characterization and analysis. IOP Conference Series: Earth and Environmental Science, v. 17, p. 012164, 2014. Disp. em: <http://stacks.iop.org/1755-1315/17/i=1/a=012164?key= crossref.2409598cc0fc31b0729a6af1955660c8>. .

YANG, Q.; LI, J.; GAN, X.; ZHANG, J.; YANG, F.; QIAN, Y. Comparison of landscape patterns between metropolises and small-sized cities: a gradient analysis with changing grain size in Shanghai and Zhangjiagang, China. International Journal of Remote Sensing, v. 33, n. 5, p. 1446-1464, 2012.

ZAMBONI, A.; HERNANDES, E.; DI THOMMAZO, A. StArt uma ferramenta computacional de apoio à revisão sistemática. In: Proc.: CONGRESSO BRASILEIRO DE SOFTWARE (CBSoft'10), Salvador, Brazil. 2010.

ZHANG, Y.; ODEH, I.O.A.; RAMADAN, E. Assessment of land surface temperature in relation to landscape metrics and fractional vegetation cover in an urban/peri-urban region using Landsat data. International Journal of Remote Sensing, v. 34 , n. 1, p. 168-189, 2013. 